\title{
Kernos
}

Revue internationale et pluridisciplinaire de religion

grecque antique

7| 1994

Varia

Simone Ruth wolf, Herakles beim Gelage. Eine motiv- und bedeutungsgeschichtliche Untersuchung des Bildes in der archaischfrühklassischen Vasenmalerei

\section{Annie Verbanck-Piérard}

\section{(2) OpenEdition}

Édition électronique

URL : http://journals.openedition.org/kernos/1126

DOI : 10.4000/kernos. 1126

ISSN : 2034-7871

Éditeur

Centre international d'étude de la religion grecque antique

Édition imprimée

Date de publication : 1 janvier 1994

ISSN : 0776-3824

Référence électronique

Annie Verbanck-Piérard, «Simone Ruth wolf, Herakles beim Gelage. Eine motiv- und bedeutungsgeschichtliche Untersuchung des Bildes in der archaisch- frühklassischen Vasenmalerei », Kernos [En ligne], 7 | 1994, mis en ligne le 16 juin 2011, consulté le 23 septembre 2020. URL : http:// journals.openedition.org/kernos/1126; DOI : https://doi.org/10.4000/kernos.1126 
Le chapitre de conclusions, intitulé "Cosmogonie, immortalité et cannibalisme», est davantage spéculatif, construit essentiellement sur l'analyse du sacrifice menée par l'«école» de Jean-Pierre Vernant. L'A. tente de replacer la cryptie dans le feu et le festin cannibalesque dans une perspective plus large, celle de la séparation cosmogonique entre hommes et dieux. Les remarques qu'elle accorde à l'opposition entre les sacrifices avec consommation des chairs mâles et les sacrifices où l'on fait simplement couler le sang des vierges sont des plus intéressants, mais sans doute un peu trop exclusives : il existe de nombreux cas de sacrifices humains où l'on se contente de faire couler le sang des garçons, et aussi quelques mythes où la chair des jeunes filles est destinée à être mangée (je pense au Minotaure et aux légendes où les vierges Andromède et Hésione sont livrées en pâture aux monstres «marins», eux aussi anthropophages; une légende obscure raconte aussi comment une jeune prêtresse fưt ébouillantée dans un chaudron à Dodone par les Thébains en guerre).

La bibliographie est complète dans sa sélectivité, mais on regrettera que les travaux de Burkert et de Meuli n'aient pas été davantage pris en considération dans la discussion. Sur Ino-Leucothéa, à côté du vieil article de Farnell, on verra plutôt C. BONNET, Le culte de Leucothéa et de Mélicerte en Grèce, au Proche-Orient et en Italie, dans SMSR, 51, 1986, p. 51-73. D'utiles annexes terminent le livre : catalogue iconographique (où n'apparaît pas la mitra d'Axos), catalogue de textes (dont les moins accessibles sont livrés en traduction française), tableaux «structuralistes" des principaux mythes, tableaux généalogiques, index des termes techniques et index général. Un livre incontournable pour toute étude relative au chaudron et au feu régénérateur dans l'Antiquité grecque.

Pierre BonNECHERE (Université de Montréal)

Simone Ruth WoLf, Herakles beim Geláge. Eine motiv und bedeutungsgeschichtliche Untersuchung des Bildes in der archaischfrühklassischen Vasenmalerei, Köln-Weimar-Wien, Böhlau Verlag, 1993. 1 vol. 21 x $28 \mathrm{~cm}$, XVII+246 p., 141 fig. (Arbeiten zur Archäologie). ISBN : 3-412-00691-2.

Au cours de ces dernières années, les recherches consacrées au banquet grec, à ses pratiques, son idéologie et ses représentations, se sont multipliées ${ }^{1}$, révélant la complexité et l'importance symbolique de

1 Quelques jalons : F, Lissarrague, Un flot d'images, Paris, 1987; O. Murray (éd.), Sympotica, Oxford, 1990 ; P. SchmitT-PANTEL, La cité au banquet, Paris, 1992. 
cette pratique constitutive de la société. Si, depuis l'ouvrage de B. Fehr notamment ${ }^{1}$, l'iconographie du banquet des "citoyens" a été souvent évoquée, il convenait certainement d'attirer l'attention sur un thème particulier et fort prometteur : celui d'Héraklès au festin, qui n'a bénéficié jusqu'ici d'aucune analyse approfondie et spécifique. C'est ce que propose S. Wolf, dans son livre Herakles beim Gelage, issu d'une Dissertation soutenue en 1988/89 à Bonn (Friedrich-WilhelmsUniversität). Le sous-titre est indispensable car il cerne d'emblée le matériel d'étude et les limites chronologiques: Eine motiv- und bedeutungsgeschichtliche Untersuchung des Bildes in der archaischfrühklassischen Vasenmalerei. Plus précisément encore, le lecteur est averti dès l'introduction (p. 5) que la céramique illustrant ce motif est essentiellement attique, la seule exception notable étant le célèbre cratère corinthien du Louvre E 635 (Héraklès chez Eurytos), qui constitue la première attestation connue.

Par un bref état de la question, l'A. réduit assez schématiquement les interprétations antérieures à l'alternative "séjour olympien ou banquet héroïque ?», notant au passage diverses hypothèses relatives aux allusions politiques, cultuelles ou théâtrales (drame satyrique). Elle définit ensuite les deux aspects de son approche: analyse typologique d'une part, analyse iconographique et iconologique d'autre part.

L'analyse typologique se fonde sur l'ensemble des vases connus représentant Héraklès couché. L'A. agence son matériel selon un classement intéressant, qui permet non seulement de déterminer des schémas distincts (au nombre de huit, outre l'analyse isolée de l'Eurytos-Krater corinthien), mais encore de souligner la primauté de certains peintres ou ateliers dans le choix et la diffusion de ces schémas. Renonçant à la distinction prônée par B. Fehr (banquet sur

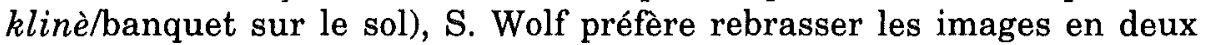
grands groupes: soit Héraklès festoie seul, même si d'autres personnages, debout ou assis, l'entourent et le regardent (type 1) ; soit Héraklès festoie avec d'autres partenaires, eux aussi couchés et "attablés": Dionysos (type 2), Hermès (type 3), Iolaos? (type 4), un hoplite ${ }^{2}$ (type $8=$

1 B. F EHR, Orientalische und Griechische Gelage, Bonn, 1971.

2 Qui est cet hoplite ? J'aurais tendance à accepter l'interprétation de l'A., qui y voit un guerrier anonyme, expression du kämpferische Tugend des Mannes (p. 158), mais la présence d'un hoplite au banquet pose problème, surtout si l'on privilégie une approche "réaliste» de l'image. Arès n'est pas à exclure aussi radicalement que le suppose l'A. : il est le frère d'Hèbè, la promise d'Héraklès. Il figure dans d'autres représentations d'Assemblées divines, où il se situe généralement, lui aussi, aux marges de l'Olympe. Le skyphos d'Athènes, M.N. 13907, donné comme inédit p. 203, sf. 30, a été très bien publié par M. PIPILI, CVA Greece 4, Athens, Nat. Museum 4, Attic Black-Figure Skyphoi, Athènes, 
"variante»). Dans ce deuxième groupe figurent également les représentations d'Héraklès entouré de satyres (type 5), ainsi que les banquets chez Pholos (type 6) et Eurytos (type 7). Cette répartition permet de mieux observer l'évolution chronologique du motif et de mieux percevoir quels peintres l'ont privilégié. Ainsi, sur le plan céramologique, il ne sera pas indifférent de savoir que le type 1 , le plus ancien et le mieux attesté, se cantonne à la figure noire (à l'exception de la splendide amphore bilingue de Munich 2301), chez les bons artistes du dernier tiers du VIe s. surtout : p. d'Antiménès, p. de Lysippidès, groupe de Léagros... Les autres variantes apparaissent dans l'ensemble vers la fin du VIe s., mais seuls les types 2 (Hér. couché avec Dionysos) et 5 (Hér. + satyres) connaissent un certain succès en figure rouge; par contre, les attestations assez nombreuses du type 3 (Hér. et Hermès) et 4 (Hér. et Iolaos ?) relèvent d'un phénomène de production en série, due l'un au peintre de Thésée, l'autre au groupe de Léagros, fait objectif dont l'interprétation demeure délicate, mais qui devrait influencer l'évaluation statistique et l'approche globale du thème. Une telle option méthodologique, appliquée dans cet ouvrage avec beaucoup de rigueur et d'esprit d'observation, me paraît particulièrement louable. En effet, on ne soulignera jamais assez, dans les enquêtes iconographiques, l'importance de l'insertion des données céramologiques les plus concrètes: il ne suffit pas de dresser un tableau chronologique général, par décennie ou quart de siècle, encore faut-il procéder à une «ventilation» des résultats par ateliers, ce qui fait parfois apparaître de véritables lignées de transmission de motifs. Si l'on tient compte du contexte artistique ponctuel (peintre, forme du vase...), le sens même d'une image peut s'en trouver nuancé, enrichi ou relativisé.

La deuxième partie, Ikonographie und Ikonologie, reprend sous un autre angle la lecture de chacun des schémas définis dans la typologie. Sont à présent examinés point par point d'une part le rapport entre les différents types du banquet hérakléen et les représentations contemporaines du banquet «humain», d'autre part les Einzelmotive, c'est-à-dire certaines composantes de la structure formelle ou certains détails de la scène, qui font l'objet d'une succession de paragraphes claire mais peu variée : analyse des attitudes, des éléments du mobilier et du "décor», des attributs, des personnages secondaires... Le type 5 (Herakles allein liegend im Besein von Satyrn) suscite bien évidemment un développement sur les rapports avec le drame satyrique, commentaire prudent et lucide que l'A. élabore notamment à partir de la belle coupe de Berlin

1993, pl. 44 et p. 51-52 (Ares or Iolaos). Pour le skyphos de Dèlos B 6138 (Dugas 595), p. 204, sf. 37, il aurait été piquant de signaler que ce vase, avec représentation d'Héraklès + hoplite (Arès ?) au banquet avait été consacré à Héra! 
F 2534 (pourtant fort tardive par rapport à l'ensemble du corpus, et qui ne représente plus un Héraklès couché). Chaque sous-chapitre donne lieu à des conclusions partielles, sur lesquelles nous allons revenir. Notons déjà que, selon $\mathrm{S}$. Wolf, le type 1 se caractérise par sa proximité visuelle avec d'autres exploits d'Héraklès : même mise en évidence du héros solitaire, "accomplissant" son festin sous le regard bienveillant de ses divinités protectrices. Par contre, les autres types, surtout diffusés à la génération suivante, sont directement influencés par les motifs et l'ambiance de l'Alltagsgelage, thème dominant des débuts de la figure rouge.

À la suite de sa prospection iconographique en deux volets principaux, typologique et iconologique, l'A. a réuni en troisième partie un dossier littéraire consacré à la Gastmahlsthematik im HeraklesMythos : nombreux sont en effet, et pour cause, les épisodes mythiques où Héraklès festoie; c'est un topos introductif commode et fréquent, qui rythme les voyages du héros. Là encore, dans la fiction du récit comme dans celle des images, $\mathrm{S}$. Wolf décèle la transposition valorisée et valorisante de pratiques réelles.

Après deux annexes consacrées l'une à la base de Lamptrai, premier témoignage sculpté attique de l'iconographie du banquet d'Héraklès, document capital qui aurait dû être mieux intégré au corps du texte, l'autre aux représentations d'Héraklès comaste, l'A. aborde son chapitre terminal, suivi du catalogue détaillé des vases étudiés (une centaine), d'index et de concordances, ainsi que d'une abondante illustration : plus de trente planches, reproduisant la presque totalité des œuvres citées, permettent de suivre, image par image, tout le raisonnement. Car la démarche a été longue et minutieuse, qui aboutit à la conclusion générale suivante : le banquet d'Héraklès - prototype du héros fort et courageux auquel peuvent s'identifier les hommes - évoque un moment de répit, calqué sur le symposion réel à une époque où celuici était le plus figuré, donc le plus significatif d'une certaine façon d'être. Le festin "à la mode d'Héraklès" est l'expression visuelle du Repos qui contrebalance les Exploits et exalte, sur le mode hérö̈que, l'alternance fondamentale de la vie aristocratique entre action d'éclat et loisir social.

Cette déduction générale, qui ne manque pas d'intérêt et semble fondée par une analyse méthodique, est effectivement logique et attendue en fonction des dossiers mis en œuvre dans ce livre bien charpenté : d'une part, et exclusivement, les vases montrant Héraklès couché, d'autre part les représentations de banquet (Alltagsgelage), prises au premier degré ; et en arrière-plan, inévitablement, les exploits familiers du Surhomme. Toutefois, était-il opportun de restreindre ainsi, dès le départ, le champ de la recherche, surtout quand il s'agit de 
l'insaisissable Héraklès attique ? N'était-ce pas s'engager là dans une forme de raisonnement tautologique?

Le fait que, dans le programme iconographique du banquet hérakléen, comme dans bien d'autres, les pratiques "réelles», ou du moins ce que les peintres nous en restituent, aient influencé, sinon inspiré, suivant un code précis et à des périodes déterminées, l'imagerie héroïque et divine, constitue une évidence, attestée également pour le thème des noces, des départs en char, de l'audition musicale, de la libation, etc. Mais cette évidence même suffit-elle pour étayer, sur base de comparaisons très ponctuelles, l'interprétation émise par l'A. ? Ou plutôt, cette évidence même, si bien mise en lumière ici, n'était-elle pas en soi le point de départ, bien plus que le point d'aboutissement d'une telle enquête ? Ce qui transparaît admirablement dans cette série d'images, l'A. l'a bien compris, c'est la plasticité du système iconographique grec, qui permet à l'artiste, sinon au spectateur, de glisser en permanence du registre humain au registre mythique et vice versa. Mais encore fallait-il, comme les peintres, explorer tous les registres... À n'aborder qu'une série limitée d'images (banquet des hommes) comme source de comparaison, le risque principal était bien de ne retenir que l'aspect «humanisé» du banquet d'Héraklès. À ne voir en Héraklès que le brave tueur de monstres des Erga et Parerga, le deuxième risque était bien de n'en déduire qu'un repos héroïque. Mais l'image n'a pas tout révélé, loin de là : elle n'a livré que ce que l'on supposait d'elle, en la regardant sous un seul point de vue.

De fait, si l'on resitue le banquet hérakléen dans l'ensemble de l'iconographie hérakléenne de cette époque, de nouvelles dimensions apparaissent qui auraient dû être suggérées, me semble-t-il. Il est impossible de faire abstraction des images archaïques et classiques, de plus en plus nombreuses et élaborées, qui mettent en scène ce que l'on peut appeler, faute de mieux, l'«Apothéose» d'Héraklès : accueil d'Héraklès par les Olympiens, rencontres, assemblées, auditions, et, plus tard, les court scenes qui le montrent en situation cultuelle, entre Athéna et Hèbè ${ }^{1}$... Cet ensemble considérable, complexe, qui fait l'objet de multiples recherches et innovations chez les peintres attiques et qui deviendra dominant au Ve s,, au détriment des Travaux, oriente bien davantage Héraklès vers le divin que vers l'héroïque. Et le festin lui aussi peut être compris comme l'une de ces formes de mise en contact d'Héraklès et des dieux, Dionysos et Hermès surtout, mais aussi Apollon, Poséidon, Arès... La contamination flagrante, mais superficielle, avec les pratiques «humaines» n'implique nullement une lecture "héroïque»: de la même façon, ce n'est pas parce qu'Athéna

1 Schéma dont les premières attestations remontent précisément au peintre de Thésée : cf. $A B V 519,18$. 
utilise un char, comme Amphiaraos ou un aurige, qu'elle perd son rang de déesse poliade. Aucun des éléments étudiés par l'A. dans la deuxième partie ne permet de définir un espace héroïque, car tel n'est pas leur rôle dans l'image : ni les couronnes, ni les fleurs, ni les phiales, ni le paysage, ni les animaux, etc. Les différents Einzelmotive peuvent avoir leur place dans un contexte humain, héroïque ou divin ${ }^{1}$ : ils ne créent pas la hiérarchie, mais assurent au contraire la confusion entre les catégories, entre "nos" catégories d'interprétation. Dès lors, une tout autre lecture est également plausible : celle de l'épiphanie divine d'Héraklès, festoyant en pleine gloire. Dans le type 1, les dieux et les hommes ${ }^{2}$ reconnaissent le nouveau statut d'Héraklès. Et dans les images où Héraklès côtoie Dionysos, ce qui me paraît le plus visible, ce n'est pas une quelconque gradation entre un héros et un dieu, mais bien la parfaite équivalence, dans les gestes et l'environnement, des deux fils de Zeus, au festin comme dans d'autres Assemblées. Je ne crois donc pas que l'on puisse retenir pour ce schéma l'expression, souvent employée par l'A., de dionysisch-heroische Bildthematik. De même, pour la coupe à fig. rouges de Florence 73127 , il s'agit bien de quatre dieux au banquet, exemple supplémentaire de l'anthropomorphisation des Olympiens, ou de l'idéalisation du quotidien, qui caractérise la production céramique attique de cette période et que cristallise la présence d'Héraklès, figure-clé de la réflexion iconographique des peintres athéniens.

Face à ces deux thèses également défendables, sommes-nous donc désormais en pleine aporie ? En réalité, le débat ne se réduit pas à un simple choix de localisation de la scène (ce que l'A. a bien compris) ni même d'identité hérakléenne. Le Festin, comme toutes les images de «Repos» d'Héraklès, ne cloisonne pas l'Alcide dans l'une ou l'autre catégorie : il s'agit bien de moments et d'espaces privilégiés où le statut d'Héraklès change. Nous assistons à l'expression de sa transmutation. L'hydrie de Londres B 301 est exemplaire à cet égard : à gauche de l'image, Alcmène, la léontè, la massue, l'épée... autant de rappels de l'activité héroïque maintenant transcendée mais toujours présente; à droite, Athéna, Hermès, la couronne : l'au-delà du dieu. Sur l'épaule,

1 Seuls les aliments et le couteau à viande pourraient être sujets à caution : mais n'indiquent-ils pas le moment du repas, plutôt que celui de la boisson, et ne désignent-ils pas Héraklès précisément comme le dieu mangeur? La question méritait d'être discutée : j'ai défendu cette hypothèse dans Herakles at Feast, in R. HÄGG (éd.), The Iconography of Greek Cult in the Archaic and Classical Period, Athènes-Liège, 1992 (Kernos, Suppl. 1), p. 85-106, en particulier 97-98.

2 La présence d'Iolaos n'est nullement incompatible avec cette affirmation : ne serait-ce pas plutôt lui qui incarne dans l'image les composantes héroïque et humaine? 
l'exploit du lion de Némée souligne le début du cycle hérakléen : le banquet n'est pas une simple étape, il est l'aboutissement ou la résultante, comme aussi sur la face principale du relief de Lamptrai.

Il n'y a certes pas lieu de démonter ici point par point, et par simple goût du paradoxe, l'argumentation de l'A. Sa thèse comprend de nombreuses observations sensibles, nuancées, utiles, sur les interférences de motifs dans l'iconographie des banquets et sur l'ambiance de paix et d'opulence qui s'en dégage. Mais je tiens simplement à mettre en garde contre une analyse unilatérale des données: les déductions proposées par S. Wolf ne me paraissent pas fausses : elles sont partielles et partiales, ou du moins prématurées, car elles ne tiennent compte ni de l'ensemble du dossier hérakléen, ni des recherches actuelles qui, parfois à propos d'autres thèmes comme la libation par exemple ${ }^{1}$, nous incitent désormais à repenser les catégories habituelles de l'imagerie religieuse grecque.

L'insuffisance d'une réflexion méthodologique préalable transparaît également dans d'autres présupposés qui n'emportent pas l'adhésion. À cet égard, les points suivants, certes difficiles et complexes, auraient mérité d'emblée une analyse plus approfondie :

- À aucun moment, l'A. ne pose véritablement la question de savoir si toutes les représentations d'Héraklès couché constituent un motif unitaire, ce qui n'est pas sûr. Par exemple, le vol par les satyres des armes d'Héraklès endormi se rattache-t-il au même réseau de signification que le banquet où Héraklès est entouré de dieux ?

- Le dossier littéraire patiemment rassemblé par $\mathrm{S}$. Wolf se révèle fort utile et plein d'enseignements. Toutefois, face aux sources iconographiques, il convenait sans doute de mieux cerner la portée des textes : leur témoignage, forcément "héroïsant» de par leur fonction narrative, ne présente qu'une similitude indirecte avec celui des vases du corpus retenu ici; il met en jeu le vaste thème d'Héraklès xenos et, s'il y a d'évidents points de jonction, les différences de perspective empêchent de parler d'une véritable "illustration": le Gastmahl ne s'identifie pas exactement au Gelage. Et faut-il rappeler que le festin couché des images à fig. noires et à fig. rouges n'est pas épique ?

- La pluralité des lectures possibles, évoquée aux p. 2 et 3 du livre, ne fait l'objet d'aucun développement. On sait pourtant à quel avenir est promis le thème du banquet en général et celui d'Héraklès en

1 A.-F. LAURENS, Intégration des dieux dans le rituel humain? L'exemple de la libation en Grèce ancienne, in Rech. et Documents du Centre Thomas More, 48 (1985), p. 35-59. Pour la méthode : F. LISSARRAGUE, L'autre guerrier, ParisRome, 1990 ; voir aussi l'ensemble des contributions de Autour de l'image, in Métis, 5 (1990). 
particulier, par le biais des reliefs votifs notamment. À cet égard, une incursion vers le domaine cultuel se révèle fort instructive ${ }^{1}$. On sait aussi combien ce motif sera réélaboré, «récupéré» sur le plan funéraire, héroïque, aristocratique, politique, eschatologique... Nombreuses sont, effectivement, les potentialités d'une telle scène, mais il ne faut pas perdre de vue que certaines sont anachroniques. Et, là encore, un dernier point risque de paraître litigieux : l'hypothèse d'un Héraklèsmiroir de la condition humaine, au destin semé de joies, de doutes et de souffrances (dans une optique d'abord aristocratique, puis de plus en plus universelle) est-elle fondamentalement de mise pour les années 530-460 ? Sans doute, mais nous n'en avons guère de preuves, et il faudrait admettre qu'un tel processus d'identification n'en est qu'à ses débuts ${ }^{2}$. Quoi qu'il en soit, je n'affirmerais pas que, dans la première moitié du Ve s., Héraklès, perçu sentimentalement, comble déjà le vide laissé par les grands dieux poliades (p. 192 :...der Moment, wo die Götter in grössere Entfernung rücken).

Ces quelques remarques n'ont d'autre but que d'inciter l'A. à poursuivre une belle recherche menée, on le devine, avec conviction et sur de bonnes bases céramologiques, afin qu'à l'avenir l'interprétation ne s'arrête plus en si bon chemin et puisse s'écarter des sentiers de la vieille tradition mythographique, trop réductrice.

Annie Verbanck-Piérard (Musée Royal de Mariemont)

\section{Notices bibliographiques}

Cahiers du GITA, 7 (1992-1993). 1 vol. 16 x 24 cm, 258 p. (Les Perses d'Eschyle, textes rassemblés par Paulette Ghiron-Bistagne, Alain Moreau, Jean-Claude TuRPIN). ISSN : 0295-9909.

Ce numéro spécial des Cahiers du Gita consacré aux Perses d'Eschyle regroupe dix communications destinées à la fois aux chercheurs et aux étudiants. Le propos est diversifié : certaines études relèvent de la philologie ou de l'histoire de l'art; d'autres s'intéressent aux rapports entre les mythes et la tragédie, ou encore à l'histoire et à la société.

1 Cf. mon article cité supra n. 5 ; j'y publie, p. 103, un fragment inédit d'un skyphos du peintre de Thésée (coll. privée suisse), à ajouter au catalogue de S. Wolf.

2 C. BÉRARD, Étrangler un lion à mains nues, in Images et société en Grèce ancienne, Cahiers d'Archéologie Romande, 36 (1987), p. 177-186. 